\title{
Validation of the Social Phobia and Anxiety Inventory for Children (SPAI-C) in a sample of Brazilian children
}

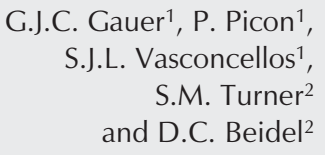

G.J.C. Gauer ${ }^{1}$, P. Picon ${ }^{1}$, S.J.L. Vasconcellos ${ }^{1}$,

S.M. Turner ${ }^{2}$ and D.C. Beidel ${ }^{2}$

\author{
'Departamento de Psiquiatria, Pontifícia Universidade Católica do Rio Grande do Sul, \\ Porto Alegre, RS, Brasil \\ 2Department of Psychology, University of Maryland, College Park, MD, USA
}

\section{Correspondence \\ G.J.C. Gauer \\ Rua Felicíssimo de Azevedo, 1455 \\ Sala 406 \\ 90640-110 Porto Alegre, RS \\ Brasil \\ Fax: +55-51-3028-9090 \\ E-mail: ggauer@terra.com.br or \\ ggauer@pucrs.br}

Presented at the 156th American Psychiatric Association Annual Meeting, San Francisco, CA, USA, May 19, 2003.

Research supported by CNPq (No. 200566/99-7). Part of a Post-Doctorate Program presented by G.J.C. Gauer to the Maryland Center for Anxiety Disorder.

Received December 5, 2003 Accepted December 14, 2004 ....................

\begin{abstract}
The purpose of the present study was to examine the factor structure and psychometric properties of the Social Phobia and Anxiety Inventory for Children (SPAI-C), an instrument developed in the United States and applied to a sample of Brazilian schoolchildren. The process included the translation of the original material from English into Portuguese by two bilingual psychiatrists and a back translation by a bilingual physician. Both the front and back translations were revised by a bilingual child psychiatrist. The study was performed using a cross-sectional design and the Portuguese version of the SPAI$\mathrm{C}$ was applied to a sample of 1954 children enrolled in 3rd to 8th grade attending 2 private and 11 public schools. Eighty-one subjects were excluded due to an incomplete questionnaire and 2 children refused to participate. The final sample consisted of 1871 children, 938 girls $(50.1 \%)$ and 933 boys (49.8\%), ranging in age from 9 to 14 years. The majority of the students were Caucasian $(89.0 \%)$ and the remainder were African-Brazilian (11.0\%). The Pearson product-moment correlation showed that the two-week test-retest reliability coefficient was $r=0.780$ and Cronbach's alpha was 0.946. The factor structure was almost similar to that reported in previous studies. The results regarding the internal consistency, the test-retest reliability and the factor structure were similar to the findings obtained in studies performed on English speaking children. The present study showed that the Portuguese language version of SPAI-C is a reliable and valid measure of social anxiety for Brazilian children.
\end{abstract}

\section{Introduction}

Social anxiety disorder (also known as social phobia) is the fear of being watched or evaluated by others. People with this disorder are extremely afraid they might say or do something that may embarrass them or expose them to criticism. As a result they tend
Key words

- Social assessment

- Social phobia

- Social anxiety

- Test reliability

- Test validity to avoid social situations or endure them with intense anxiety or distress (1).

Although mid-adolescence had been considered to be the average age of onset, recent studies have shown that children as young as eight meet diagnostic criteria for the disorder (2-4). Furthermore, social anxiety disorder can result in immediate and long-term impairment 
involving many aspects of daily functioning and can prevent the achievement of future social and professional development in these children (4). Nevertheless, social anxiety disorder continues to be largely ignored in clinical practice, and it is quite likely that many clinicians do not take it as seriously as they should. This is particularly unfortunate because, in the great majority of cases, social anxiety disorder in children responds extraordinarily well to cognitive behavioral therapy and psychopharmacotherapy (5-9). It is also important to remember that the disorder should be identified as early as possible, before it begins to jeopardize the potential of these children. In addition, when assessing children the clinician should be aware of some differences in the clinical presentation of the symptoms when compared with adults. For instance, in children there must be evidence of a capacity for social relationships with familiar people and the anxiety must occur in the peer settings, not just in interaction with adults, or during public performance, for example (4). Therefore, it is really important to have reliable instruments during the disorder evaluation process.

The Social Phobia and Anxiety Inventory for Children (SPAI-C) has shown initial promise in the assessment of distress in a variety of social situations (10). Nevertheless, until now investigations have focused on North American populations, and the test must be validated in other languages and cultures. The current study was designed to validate SPAI-C in a different cultural group in order to make this instrument accessible to clinicians and researchers in the Brazilian Portuguese language.

\section{Material and Methods}

\section{Social Phobia and Anxiety Inventory for Children}

SPAI-C is an empirically derived inventory developed specifically to assess social phobia in childhood and early adolescence, as a screening scale (10). Useful in clinical and research settings, the SPAI-C assesses a range of potentially anxiety-producing situations (reading aloud, performing in a play, and eating in the school cafeteria) and assesses physical and cognitive characteristics of social phobia as well as avoidance behaviors. Each of the 26 items is rated on a 3point scale (never or hardly ever, sometimes, most of the time or always). Some of the items require multiple responses. SPAI-C uses a Likert scale format that allows an assessment of the frequency with which each symptom is experienced. The maximum score on SPAI-C is 52, which indicates that the child experiences anxiety with a high frequency in a broad range of social settings. In addition to its quantitative score, the scale can be used qualitatively to examine different patterns of responses to various types of social situations (10). Such an examination may assist the clinician in determining the salient aspects of the child's fear, and thus may be important for the development of an appropriate treatment plan. In the initial investigation, SPAI-C had high internal consistency $(\alpha=0.95)$, high 2 -week test-retest reliability $(r=0.86)$, and adequate reliability at 10 months $(r=0.63)(10)$. After this initial investigation, other studies have been conducted to determine the reliability and validity of SPAI-C (11).

\section{Instrument translation}

The SPAI-C Portuguese validation process, authorized by its authors through MultiHealth Systems Incorporation (USA) included the translation of the original material from English into Portuguese by two bilingual psychiatrists and a back translation by a bilingual physician. Both the front and back translations were revised by a bilingual child psychiatrist. Finally, all the front and back translations were sent to be analyzed by the authors of the instrument. 


\section{Sample}

The initial sample consisted of 1954 Brazilian schoolchildren enrolled in the $3 \mathrm{rd}$ to the 8 th grade attending 2 private and 11 public schools in the metropolitan area of Porto Alegre, the southernmost state capital in the country.

After contacting the school directors and offering a presentation of the project to the teachers, the sample process was started. All students aged 9-14 years were chosen to participate. In some cases, the parents had given informed consent for the child to participate, as required by some school principals. Hence, the sample was not randomly selected but rather included all available students. The research assistant reported that only two children refused to fill out the questionnaire and that 81 subjects were excluded because they failed to complete it. The final sample consisted of 1871 children, 938 girls $(50.1 \%)$ and 933 boys (49.8\%). The sample ranged in age from 9 to 14 years (mean $\pm \mathrm{SD}=12.26 \pm 1.38$ years $)$. The majority of the students were Caucasian $(89.0 \%)$ and the remainder were AfricanBrazilian (11.0\%). Most $(97.0 \%)$ were born in the state and only a few $(3.0 \%)$ came from other regions of the country. The sample represented a wide range of socioeconomic levels.

Two weeks after the initial administration, SPAI-C was readministered to $410 \mathrm{sub}$ jects of the original sample. This second sample consisted of 187 boys (45.6\%) and 223 girls $(54.4 \%)$ chosen according to convenience criteria, i.e., schools with a larger number of students. The purpose was to reach a sample size of at least 200 children to perform an adequate test-retest reliability study (12).

\section{Assessment}

The total length of time necessary to collect the test and retest data was 3 months.
After being trained to apply the scale, 16 research assistants administered the questionnaire in 86 classrooms in the metropolitan area. In each classroom, students completed the self-report measure as a group after receiving instructions on how to proceed. For young children, especially those in the 3rd or 4th year, the research assistant read the questions aloud. Research assistants circulated among the students during the test session and provided individual help to any student who experienced difficulty. Completion of the SPAI-C took approximately $45 \mathrm{~min}$. After the students had completed the questionnaire, the research assistant reviewed them and if anything was missing or a mistake was detected, the questionnaire was returned to the children and they were encouraged to complete it. Since the students were not required to indicate their name and identification number in the questionnaire, they could not be identified after they completed the application. In order to be able to compare the test with retest, the research assistants gave a number to the students who were chosen to participate in the second application.

\section{Statistical analysis}

A reliability study was performed, which included the internal consistency analysis (Cronbach's alpha) of the Portuguese language instrument using the total sample of 1871 children, and test-retest reliability was calculated (using Pearson's r) with a Portuguese language instrument version in a subsample of 410 school students, with a twoweek break between assessments.

The factor structure of the 26-item SPAI$\mathrm{C}$ was examined using factorial analysis validation with a varimax rotation of data with the Portuguese language instrument version assessed in the total sample.

The Student $t$-test was used to analyze the differences of the test results between sex groups and race groups. 


\section{Results}

\section{Descriptive characteristics of SPAI-C}

For the total sample, the minimum score was 0 and the maximum 50. For the group of 938 girls, the minimum score was 0 , the maximum 50 and the mean $( \pm$ SD) was 16.90 \pm 8.51 . For the group of 933 boys, the minimum score was 0 , the maximum was 42 , and the mean was $14.85 \pm 8.12$. The difference between boys and girls was significant $(\mathrm{P}<$ 0.001 ; Student $t$-test). The mean for the total sample was $15.88 \pm 8.38$ for the test and $13.92 \pm 8.67$ for the retest.

For the group of Caucasian children, the minimum score was 0 and the maximum 50, and the mean was $15.76 \pm 8.35$. For the group of African-Brazilian children, the minimum score was 0 and the maximum 41, and the mean was $16.86 \pm 8.51$. There was no difference in total score according to race at the 0.05 level of significance $(\mathrm{P}=0.075$; Student $t$-test).

\section{Internal consistency}

Intrascale reliability was calculated by Cronbach's alpha reliability estimates. The

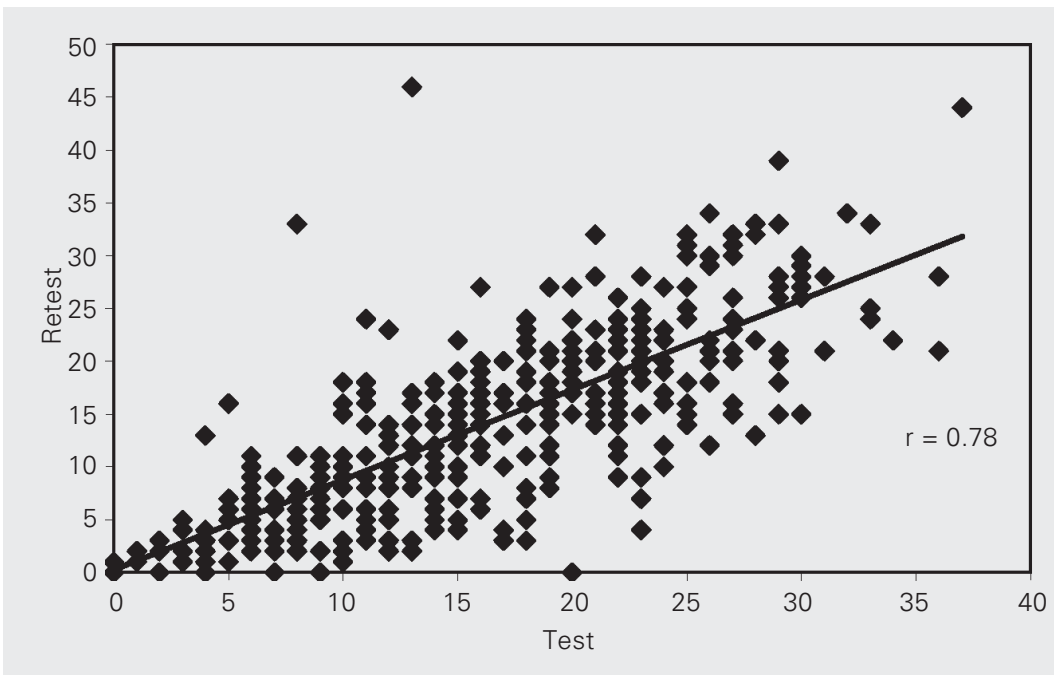

Figure 1. SPAI-C: Scatter plot of test and retest values, least squares line and the Pearson product-moment correlation. The test was taken by 1871 students and the retest by 410 students 14 days later. Data are reported for students who took both tests.
SPAI-C reliability for the entire sample was 0.94. Reliability was 0.94 for each of the groups, i.e., boys and girls and African-Brazilian and Caucasian children separately.

\section{Test-retest reliability}

The test-retest reliability was calculated using the Pearson product-moment correlation. For the total sample of 410 children the two-week test-retest reliability coefficient was $r=0.78$, as shown in Figure 1. For both sexes as a group the two-week test-retest reliability coefficient was $r=0.77$ for the boys and $r=0.78$ for the girls. The two-week test-retest reliability coefficient was $r=0.78$ for African-Brazilian children as a group and $\mathrm{r}=0.77$ for Caucasian children.

\section{Factor analysis}

The factor structure of the 26-item SPAI$\mathrm{C}$ was examined using factor analysis validation with a varimax rotation of data in the Portuguese version of the instrument assessed in the 1871 children. There were four factors with eigenvalues higher than 1 . Only items that loaded 0.40 or greater on a factor were retained within a factor. Using this criterion, only one item failed to load on a factor. Taken together, these factors accounted for $47.66 \%$ of the total variance. The items and their factor loadings are presented in Table 1.

The first factor was labeled Assertiveness and accounted for $13.90 \%$ of the variance. The second factor was labeled Avoidance/ Social Encounters and accounted for $11.99 \%$ of the variance. The third factor was labeled Public Performance and accounted for $11.74 \%$ of the variance. The fourth factor was called Physical and Cognitive Symptoms and accounted for $10.03 \%$ of the variance.

\section{Discussion}

The results of internal consistency and the test-retest reliability were similar to the 
findings in studies performed with children in areas where English is spoken. The data on internal consistency and test-retest reliability obtained by this investigation showed satisfactory coefficient alpha values and Pearson product-moment correlation, confirming the good psychometric properties of the instrument found in previous studies.

The factor structure was almost similar, but not identical, to that reported in two previous studies performed with children in the United States $(10,11)$. We detected Assertiveness as a first factor, similar to that observed in previous studies but, in contrast to these studies, we did not find the General Conversation factor. As factor number two we found Avoidance/Social Encounters. It is interesting that the items included in this factor are similar to the Traditional Social Encounters factor found in the first study, and to the Avoidance factor found in the second study, but not in the first. Factor three, Public Performance, was similar to both previous studies. Factor four, Physical and Cognitive Symptoms, on the other hand, was found in the second study but not in the first. Thus, most factors that were found in the previous studies were also found in the present study. Only the General Conversation factor was not found, nor any new factor. These small differences are perhaps due to the fact that the previous studies mixed small samples of patients and normal controls whereas we conducted an epidemiological study on a non-clinical sample.

The fact that the girls scored significantly

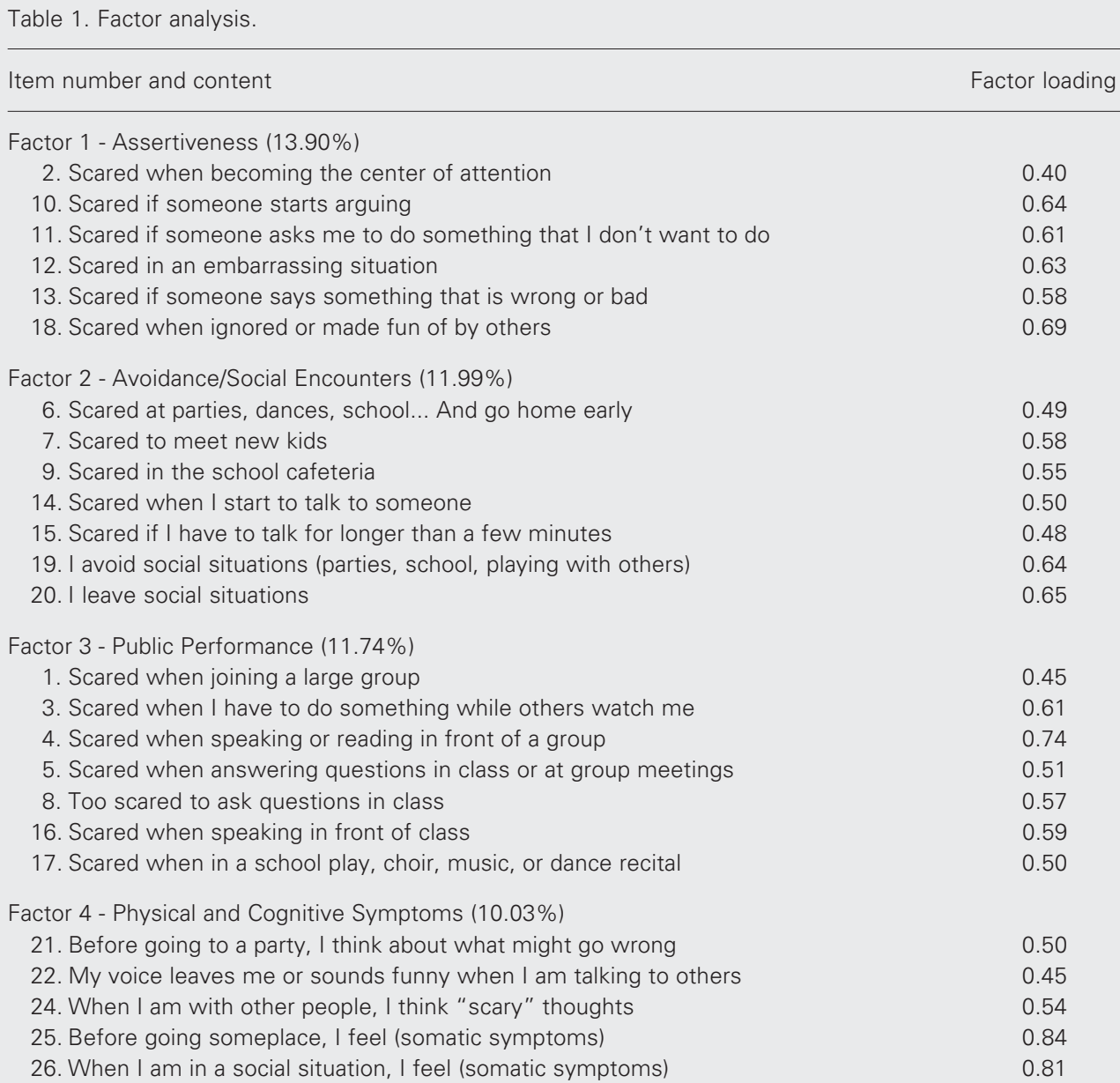


higher than the boys confirms the previous studies that also found the same differences between sexes (4). On the other hand, the scores achieved by the 205 African-Brazilian children were not significantly different from the scores achieved by the 1666 Caucasian children. The American studies found that social phobic African-American children scored significantly lower than Caucasian children (4). Perhaps the difference between the American and the Brazilian studies is a consequence of our non-clinical sample. Therefore, we believe that this matter should be better investigated before we can arrive at a more definite conclusion.

Some limitations of the present investigation should be noted. First, the extrapolation of the present findings to clinical samples is limited by the non-clinical nature of the present sample. Second, the construct validity of SPAI-C with other measures of social anxiety (convergent validity) and the comparison of social phobic children with other disorders (discriminant validity) must be evaluated for this population. Assessing an instrument's construct validity is a constant process requiring multiple efforts over time. Despite the fact that we still have a few more steps to go, the results of the present study have shown that the Brazilian Portuguese language version of SPAI-C is a reliable and valid measure of social anxiety for Brazilian children and, because of its appropriate psychometric properties, it can be used in clinical as well as in research settings.

\section{References}

1. American Psychiatric Association (2000). Diagnostic and Statistical Manual of Mental Disorders. 4th edn. Text Revision. American Psychiatric Press, Washington, DC, USA.

2. Beidel DC (1991). Social phobia and overanxious disorders in school age children. Journal of the American Academy of Child and Adolescent Psychiatry, 30: 545-552.

3. Francis G, Last CG \& Strauss CC (1992). Avoidant disorder and social phobia in children and adolescents. Journal of the American Academy of Child and Adolescent Psychiatry, 31: 1086-1089.

4. Beidel DC \& Turner SM (1998). Shy Children, Phobic Adults: Nature and Treatment of Social Phobia. American Psychological Association Books, Washington, DC, USA.

5. Nathan PE \& Gorman JM (1998). A Guide to Treatments that Work. Oxford University Press, New York.

6. Schneier FR (1999). Extreme fear, shyness, and social phobia: treatment and intervention. In: Schimidt LA \& Schulkin J (Editors), Extreme Fear, Shyness, and Social Phobia. Oxford University Press, New York

7. Beidel DC (1998). Social anxiety disorder: Etiology and early clinical presentation. Journal of Clinical Psychiatry, 59: 27-31.

8. Kaminer D \& Stein DJ (1999). Social anxiety disorder in children and adolescents. In: Westenberg HGM \& den Boer JA (Editors), Focus on Psychiatry: Social Anxiety Disorder. Syn-Thesis, Amsterdam, The Netherlands.

9. Wagner KD (2003). Paroxetine treatment of mood and anxiety disorders in children and adolescents. Psychopharmacology Bulletin, 37: 167-175.

10. Beidel DC, Turner SM \& Morris TL (1995). A new inventory to assess social anxiety and phobia: The Social Phobia and Anxiety Inventory for Children. Psychology Assessment, 7: 73-79.

11. Beidel CD, Turner SM \& Fink CM (1996). Assessment of childhood social phobia: construct, convergent, and discriminative validity of Social Phobia and Anxiety Inventory for Children (SPAI-C). Psychology Assessment, 8: 235-240.

12. Streiner DL \& Norman GR (1995). Health Measurements Scales: A Practical Guide to their Development and Use. 2nd edn. Oxford University Press, Oxford, UK. 